\title{
Hippocampal Place Cells, Context, and Episodic Memory
}

\author{
David M. Smith* and Sheri J.Y. Mizumori
}

\begin{abstract}
Although most observers agree that the hippocampus has a critical role in learning and memory, there remains considerable debate about the precise functional contribution of the hippocampus to these processes. Two of the most influential accounts hold that the primary function of the hippocampus is to generate cognitive maps and to mediate episodic memory processes. The well-documented spatial firing patterns (place fields) of hippocampal neurons in rodents, along with the spatial learning impairments observed with hippocampal damage support the cognitive mapping hypothesis. The amnesia for personally experienced events seen in humans with hippocampal damage and the data of animal models, which show severe memory deficits associated with hippocampal lesions, support the episodic memory account. Although an extensive literature supports each of these hypotheses, a specific contribution of place cells to episodic memory has not been clearly demonstrated. Recent data from our laboratory, together with previous findings, indicate that hippocampal place fields and neuronal responses to task-relevant stimuli are highly sensitive to the context, even when the contexts are defined by abstract task demands rather than the spatial geometry of the environment. On the basis of these findings, it is proposed that place fields reflect a more general context processing function of the hippocampus. Hippocampal context representations could serve to differentiate contexts and prime the relevant memories and behaviors. Since episodic memories, by definition, include information about the time and place where the episode occurred, contextual information is a necessary prerequisite for any episodic memory. Thus, place fields contribute importantly to episodic memory as part of the needed context representations. Additionally, recent findings indicate that hippocampal neurons differentiate contexts at progressively finer levels of detail, suggesting a hierarchical coding scheme which, if combined with temporal information, could provide a means of differentiating memory episodes. ๑ 2006 Wiley-Liss, Inc.
\end{abstract}

KEY WORDS: hippocampus; context; discrimination; learning; spatial

\section{INTRODUCTION}

The hippocampus has been the target of intense scrutiny since the 1957 report on the remarkable case of patient H.M., who became severely amnesic following bilateral medial temporal lobectomy (Scoville and Milner, 1957). In the intervening $50 \mathrm{yrs,} \mathrm{a} \mathrm{vast} \mathrm{literature} \mathrm{on} \mathrm{hippo-}$ campal function has accumulated. Although there is near unanimous agreement that the hippocampus is critically involved in learning and memory functions, there remains considerable debate over the precise contribution of the hippocampus to these functions.

\footnotetext{
Department of Psychology, University of Washington, Seattle, Washington

Grant sponsor: NIH; Grant numbers: MH67399, MH58755.

*Correspondence to: David M. Smith, Ph.D., Department of Psychology, Campus Box 351525, University of Washington, Seattle, WA 98195, USA. E-mail: dmsmith4@u.washington.edu

Accepted for publication 10 June 2006

DOI 10.1002/hipo.20208

Published online 8 August 2006 in Wiley InterScience (www.interscience. wiley.com).
}

Early attempts to duplicate the amnesia seen in H.M. in animals using conditioning tasks were largely unsuccessful. However, two major discoveries fostered different animal models, which have had a profound impact on the direction of research on hippocampal function. The first was the discovery that hippocampal neurons exhibit spatially localized firing patterns when rats explore their environment (O'Keefe and Dostrovsky, 1971). The second was the discovery that medial temporal lobe damage caused a severe impairment in object recognition memory in monkeys, as measured by the delayed nonmatching to sample (DNMS) task (Mishkin, 1978). These discoveries established two more or less independent lines of research focused on the cognitive mapping and declarative memory roles of the hippocampus. With a few notable exceptions (see Hippocampus, vol. 9(4), 1999), these two lines of research have been pursued more or less independently.

The recognition memory impairment seen in the DNMS task, combined with spared function in a variety of other forms of memory (e.g., motor skill learning and simple conditioning) led to the idea that the hippocampus is specifically involved in memory for facts and events, referred to as declarative memory (Squire and Zola-Morgan, 1991; Cohen and Eichenbaum, 1994; Eichenbaum and Cohen, 2001). However, more recent reports have indicated that subjects with hippocampal damage can exhibit preserved memory for facts (Vargha-Khadem et al., 1997; but see Squire et al., 2004). This has led proponents of the declarative memory hypothesis to adopt the memory structure proposed by Tulving (1972). This organizational structure holds that episodic memory, which refers to memory for personally experienced events, is distinct from semantic memory, which involves memory for facts and general knowledge of the world. Episodic memory is a particularly rich form of memory, which typically includes information about the events that comprise the episode, the people and objects that were present, the place or context in which the episode occurred, and temporal information that provides a sense of chronology. According to the revised formulation of the declarative memory hypothesis, the hippocampus is needed for episodic memory but not for semantic memory (Tulving and Markowitsch, 1998).

The discovery of hippocampal place cells has been equally influential, having fostered a remarkably extensive and detailed literature on the hippocampal role in spatial navigation (for reviews see McNaughton et al., 1996; O’Keefe, 1976; O’Mara, 1995; Muller et al., 1996; 
Wiener, 1996; Mizumori et al., 1999). The initial finding of spatially localized firing patterns was interpreted as suggesting that the hippocampus is involved in generating a mental representation of the spatial layout of the environment, known as a cognitive map (O'Keefe and Dostrovsky, 1971). This idea was supported by numerous reports indicating that hippocampal neurons are exquisitely sensitive to the spatial geometry of the environment (e.g., Kubie and Ranck, 1983; Muller and Kubie, 1987b; O'Keefe and Burgess, 1996a; Gothard et al., 1996) and findings of spatial learning impairments resulting from hippocampal lesions (Olton et al., 1979; Morris et al., 1982; Sutherland et al., 1983; Eichenbaum et al., 1990). These findings formed a highly coherent body of data consistent with the idea that the hippocampus is primarily involved in generating cognitive maps. However, many findings have indicated that hippocampal neuronal response patterns are sensitive to nongeometric features of the recording situation. For example, hippocampal neurons exhibit changes in their spatial firing patterns (i.e. place fields) with changes in colors and odors associated with an environment (Anderson and Jeffery, 2003; Hayman et al., 2003), task demands (Markus et al., 1995; Wood et al., 2000; Song et al., 2005; Smith and Mizumori, 2006), and even the problem solving strategies used by the subjects (Yeshenko et al., 2001). These findings indicate that spatial geometry cannot be the sole determinant of hippocampal neuronal responses.

\section{THE HIPPOCAMPAL ROLE IN CONTEXT PROCESSING}

In the 1970s, a third theory of hippocampal function emerged from studies of Pavlovian and instrumental learning (Hirsh, 1974; Winocur and Olds, 1978). This theory holds that the hippocampus is involved in processing the background 'contextual' information present in any learning situation. This theory has not received the attention that the episodic memory and spatial navigation accounts have. Nevertheless, an extensive literature involving experimental brain lesions has accumulated, which demonstrates that the hippocampus is critically involved in context processing (for reviews see Myers and Gluck, 1994; Maren, 2001; Anagnostaras et al., 2001). For example, hippocampal lesions impair conditioned fear responses to contextual stimuli (Kim and Fanselow, 1992; Phillips and LeDoux, 1992) and lesions of the hippocampus or entorhinal cortex (EC) render subjects insensitive to changes in the context (Penick and Solomon, 1991; Freeman et al., 1997).

A recent study examined the importance of hippocampal output to cingulate cortical and anterior thalamic brain regions during contextual learning (Smith et al., 2004). This study took advantage of the well-documented role of the cingulate cortex and anterior thalamus in instrumental discrimination learning (Gabriel, 1993) and examined whether hippocampal output modulates functioning in these regions in a context-dependent manner. Subjects with fornix lesions, which partially disconnect the hippocampus from the cingulate cortex and anterior thalamus, were trained on two different instrumental discrimination tasks, each of which was presented in a different context. Subjects with lesions were severely impaired in learning the two context-specific discrimination tasks. Control subjects developed context-specific neuronal response patterns in the cingulate cortex and anterior thalamus. However, these contextspecific neuronal responses were degraded in subjects with lesions. These results suggested that the hippocampus generates a neural representation of the context, which can be transmitted to extrahippocampal brain regions to facilitate contextual modulation of behavioral responses and memories.

\section{WHAT CONSTITUTES A CONTEXT?}

The previously described research defined the context in terms of the continuously present background cues. However, a number of findings suggest that this does not adequately describe the hippocampal role in context processing. For example, hippocampal neurons exhibit responses to foreground cues, such as the conditional stimuli and reinforcing stimuli used in conditioning tasks (e.g., Solomon et al., 1986; Eichenbaum et al., 1987; Kang and Gabriel, 1998; Moita et al., 2003). Moreover, subtle changes in task demands are associated with striking changes in the spatial firing patterns of hippocampal pyramidal neurons even though the background environment was unchanged (Markus et al., 1995; Skaggs and McNaughton, 1998; Wood et al., 2000; Song et al., 2005). Recent studies from our laboratory have shown that explicitly manipulating the behavioral and mnemonic demands of the task or the problem solving strategy also lead to highly differentiated neuronal firing patterns (Yeshenko et al., 2001; Smith and Mizumori, 2006). The hippocampus has even been implicated in selecting behavioral responses on the basis of subjects' internal motivational state (Kennedy and Shapiro, 2004).

Thus, the data indicate that hippocampal neuronal responses differentiate all of these different aspects of a learning situation. We suggest that hippocampal neurons exhibit differential firing patterns whenever subjects must distinguish one situation from another to retrieve the correct behavioral responses or memories. This idea is consistent with a broader, common usage definition of context as "the interrelated conditions in which something exists or occurs" (Merriam-Webster Collegiate Dictionary, 10th Ed.). Context, as we will use the term in the following discussion, refers to a particular situation or set of circumstances that must be differentiated from other situations in order for subjects to retrieve the correct behavioral or mnemonic output. Importantly, this definition encompasses the traditional definitions of context used historically in psychology (i.e., the background stimuli) as well as the kinds of contexts that are identified by more abstract features, such as the task demands.

\section{NEUROPHYSIOLOGICAL EVIDENCE} OF HIPPOCAMPAL CONTEXT PROCESSING

The above definition of the context was incorporated into a recent experiment, which required subjects to distinguish two 
contexts that differed only in terms of their behavioral requirements (Smith and Mizumori, 2006). In this experiment, rats were trained to retrieve rewards from one location on a plus maze during the first half of each training session and from a different location in the same environment during the second half of the sessions. Specifically, the rats were given daily training sessions during which the reward was always placed at the end of the east arm for the first 15 trials and was always placed at the end of the west arm during the second 15 trials. The start position for each trial was randomly selected from the three nonrewarded arms. In between trials, the rats were placed on an intertrial interval (ITI) platform adjacent to the maze. The position of the ITI platform was constant throughout training. The rats were given identical training sessions each day until they reached a behavioral criterion of $75 \%$ correct choices. The two session halves constituted separate contexts defined by their differing task demands. In each block of trials, the rats had to remember and approach a different reward location. Neuronal responses were examined to determine whether they differed in the two contexts.

As a control condition, the rats were given a random reward training session before beginning regular training sessions. During the random reward session, the rats started each trial on a randomly designated arm and searched for rewards located on a different randomly designated arm. The random reward session was also divided into 2 blocks of trials, although all of the trials consisted of searching for randomly placed rewards, and the neuronal responses were compared across these blocks. Since the task demands did not differ across these 2 blocks, there was no context manipulation and the neuronal responses were not expected to differ.

Learning to distinguish the two contexts was associated with the development of highly differentiated spatial firing patterns (Figs. 1A-D). These firing patterns became significantly more distinct after learning, relative to the random reward control condition. Importantly, the differential responses developed only in rats that were given context training and not in rats that were given repeated random reward sessions, indicating that the context specific place fields could not have been due to factors unrelated to learning to distinguish the contexts. The differential responses could not be attributed to differences in the rats' direction of travel in the two contexts, since the firing patterns remained significantly different in the two contexts when direction was controlled. The place fields did not simply rotate $180^{\circ}$, as would be expected if the neurons fired in relation to the reward locations, or if the firing occurred in relation to the rat's position along a given path (e.g., an inbound run, followed by a right turn and an outbound run), a phenomenon known as path equivalence (Frank et al., 2000).

As mentioned previously, hippocampal neurons are known to exhibit responses to task relevant events and stimuli (e.g., Eichenbaum et al., 1987; Kang and Gabriel, 1998; Moita et al., 2003). In our studies, the neurons developed remarkably different responses to the reward and the beginning of the ITI period in the two contexts (Fig. 1F-I). As was the case with the place fields, these responses developed as the rats learned to differentiate the contexts and they did not develop in rats that were not given context training.

Consistent with previous studies (Frank et al., 2000; Wood et al., 2000; Ferbinteanu and Shapiro, 2003), many of the neuronal responses we observed were contingent upon complex conjunctions of events. For example, some neurons exhibited place fields only when the rat arrived at the location from a particular start position (Fig. 1E). Reward responses could also be contingent on a given start position. These complex responses will be discussed further in later sections. The point to be made here is that even these complex response patterns depended on the context.
FIGURE 1. Context-specific neuronal firing patterns. Contour plots (A-D) illustrating the spatial firing patterns during the random reward session and asymptotic performance sessions. The regions of the maze visited by the rat are outlined in white. The firing rates are illustrated by the height and color of the contour peaks, with the scale indicated for each neuron. Plot A illustrates the firing pattern of a neuron recorded during the first and second halves of the random reward session (Block 1 and Block 2). For each trial, rewards were placed at the end of randomly designated arms, and the rat started at one of the three nonrewarded arms. Plots B-D illustrate the context-specific firing patterns of neurons recorded during asymptotic performance. Each pair of plots illustrates neuronal firing during the first half of the session (Context A) when the reward was always placed on the east arm, and during the second half (Context B) when the reward was always placed on the west arm. The firing patterns were similar across the two blocks of trials of the random reward session (A) but they were markedly different in the two blocks of trials (contexts) during asymptotic performance (B-D). Spike waveform overlays from both wires of the stereotrodes recorded during each half session and pixel by pixel spatial correlation coefficients $(r)$ are given for each pair of plots. Plot $E$ illustrates the spatial firing patterns of a neuron that exhibited responses to complex conjunctions of locations and events. Plots that included the data of all of the trials (Context A: All and Context B: All), regardless of the start position, show that the neuron exhibited a place field on the east arm during performance in Context $A$ and a field on the west arm in Context $B$. The same data are shown in separate firing rate maps for trials that started from the north, south, east, and west arms. The firing on the east arm in Context A occurred primarily on trials, which started from the south arm. The firing on the west arm in Context B occurred primarily on trials, which started on the east arm, opposite the reward. Peri-event time histograms illustrating event related firing of individual neurons recorded during asymptotic performance are shown in plots F-I. For each plot, the firing was summed across the 15 trials of each training block (context) with raster displays illustrating the trial by trial neuronal firing (one row of tick marks per trial). Twenty seconds of data are shown, from $10 s$ before to $10 s$ after the event. Examples of neuronal responses at the time of the reward ( $F$ and $G$ ) and the arrival at the intertrial interval (ITI) platform after training trials ( $\mathrm{H}$ and I) are shown. The firing patterns during the first half of the session (Context A), when the reward was always placed on the east arm, were markedly different from firing during the second half (Context B), when the reward was always placed on the west arm. 
Random Foraging
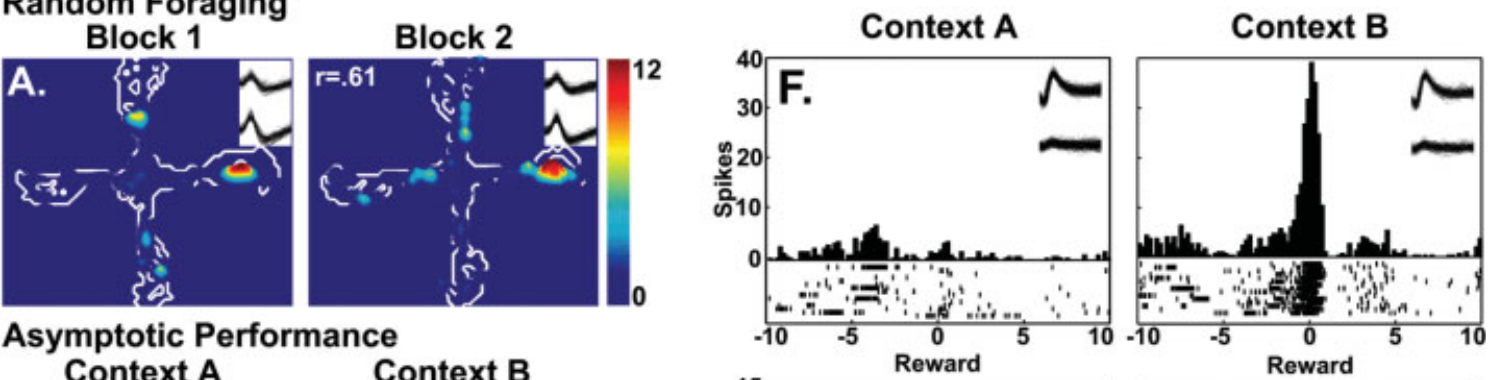

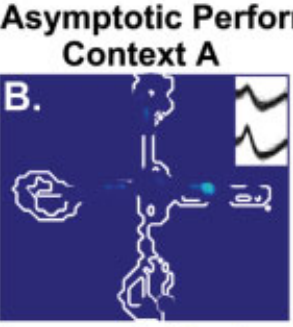

Context B
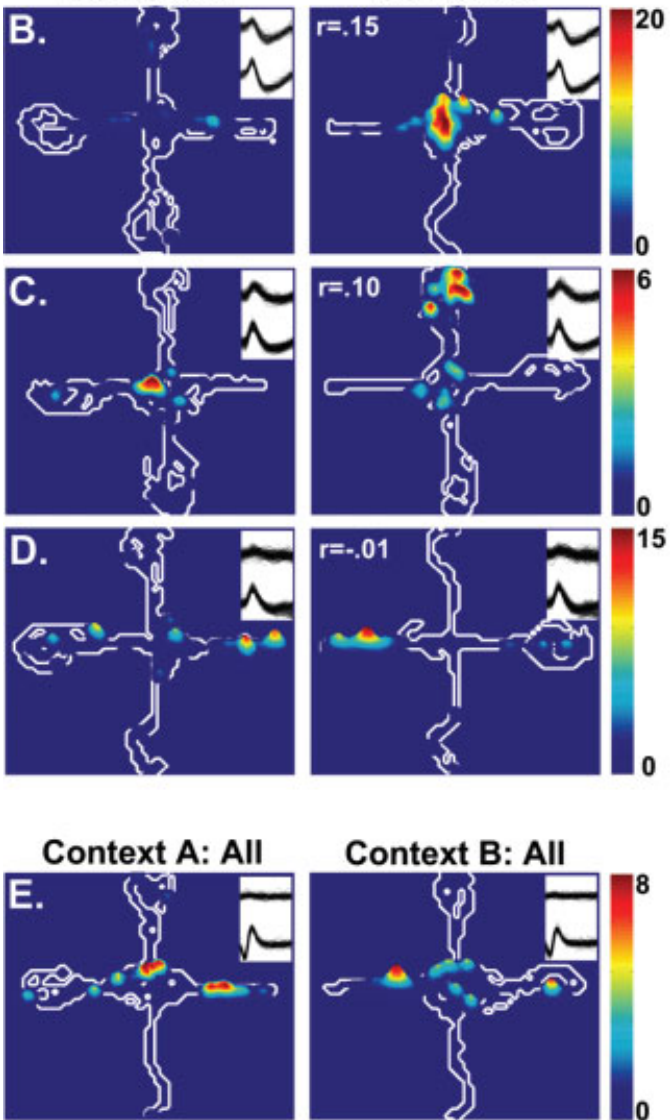

Context B: All

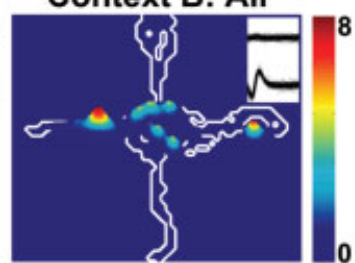

Context A: North

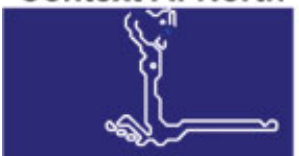

Context B: North

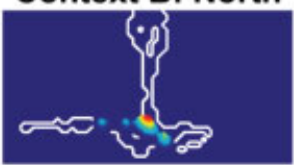

Context A: West
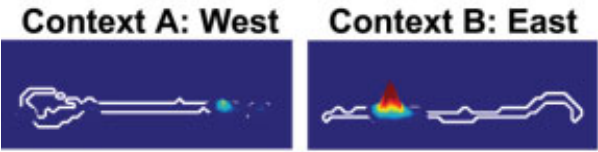

Context A: South

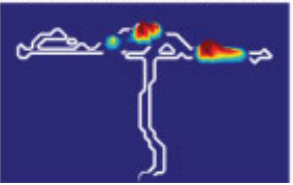

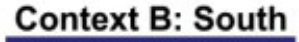

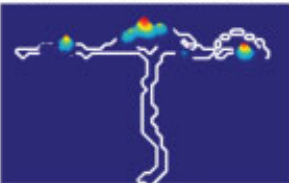

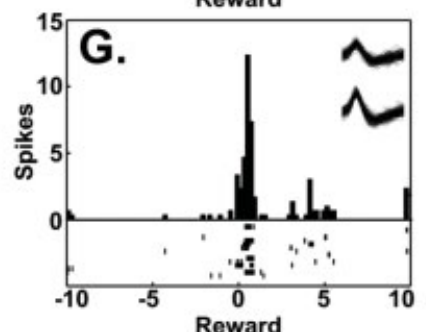
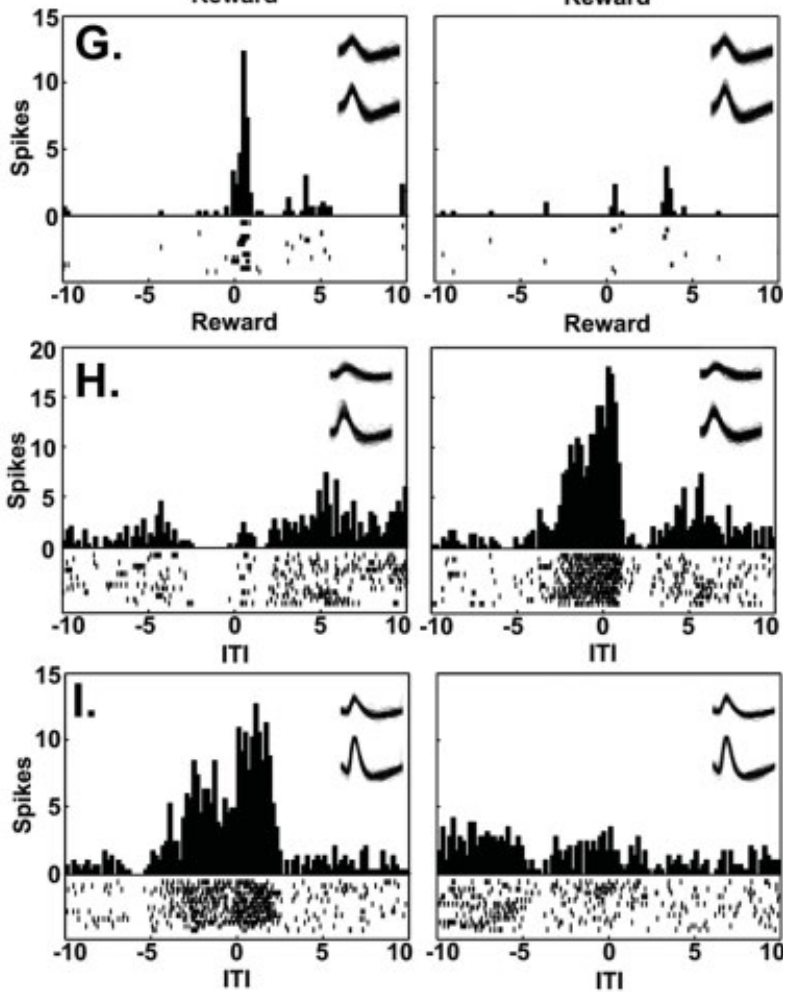

FIGURE 1 
The firing patterns of hippocampal neuronal populations were unique to each context and could therefore serve as a neural representation of the context. Context representations play a critical role in learning and memory because they provide a cue that primes the context-appropriate memories and behaviors (Blanchard and Blanchard, 1972; Godden and Baddely, 1975; Balaz et al., 1980; Fanselow, 1986). When a subject encounters a known context, the hippocampal context code is expressed and the appropriate memories and behaviors are primed. For example, the neuron in Figure 1C fired on the north start arm in the Context B but not in Context A. The firing of this neuron and others like it could prime the "right turn" response needed to reach the goal location in Context B. When these neurons do not fire, the right turn response may be suppressed while other neurons presumably prime the left turn response appropriate to Context A. In this way, the hippocampus could bias the behavioral expression systems of the brain (Mizumori et al., 2004). These results support context processing accounts of hippocampal function (Hirsh, 1974; Penick and Solomon, 1991; Kim and Fanselow, 1992; Phillips and LeDoux, 1992; Freeman et al., 1997) and they join a growing body of data that has led to a recent resurgence of interest in the context processing role of the hippocampus (Chun and Phelps, 1999; Mizumori et al., 1999; Rosenbaum et al., 2001; Jeffery et al., 2004; Johnson, 2004; Smith et al., 2004; Weis et al., 2004; LaBar and Phelps, 2005; Shanks et al., 2005).

\section{HIPPOCAMPAL INACTIVATION IMPAIRS CONTEXTUAL LEARNING}

The idea that hippocampal lesions impair context processing is well documented (Penick and Solomon, 1991; Kim and Fanselow, 1992; Phillips and LeDoux, 1992; Freeman et al., 1997; Smith et al., 2004). To determine whether learning the above described context discrimination task depended on the hippocampus, the $\mathrm{GABA}_{\mathrm{A}}$ agonist muscimol $(0.5 \mu \mathrm{g}$ in $0.5 \mu \mathrm{l}$ saline in each hemisphere) was used to temporarily inactivate the dorsal hippocampus before the initial two training sessions. Consistent with previous reports (Eichenbaum et al., 1990; Bunsey and Eichenbaum, 1996; Whishaw and Tomie, 1997; Buckmaster et al., 2004), the loss of hippocampal processing was associated with highly inflexible behavioral strategies. Many of the rats that were given muscimol simply adopted the strategy of always turning right or always turning left. This caused those rats to make many errors from some start positions but few errors from others. For example, because the reward was on the east arm in context $\mathrm{A}$, a rat that always made right turn responses would always perform correctly from the south arm but would always make an error from the north arm. In contrast, rats that did not adopt inflexible strategies would be no more likely to make errors from one start position than another. To examine this, the difference in the probably of making an error from different start positions was computed and compared across groups. High values indicated an inflexible strategy. This measure of behavioral inflexibility was signifi- cantly greater in subjects that were given muscimol than in controls that were given saline solution $(\mathrm{F}[1,7]=7.61, P<$ 0.05).

\section{PLACE FIELDS ARE PART OF A CONTEXT REPRESENTATION}

This special issue of Hippocampus is focused on the question of how hippocampal place cells are related to episodic memory. In the following discussion, we propose that place cells participate in episodic memory processes by providing a neural representation of the context, which is a necessary component of episodic memories. Before doing so, it is important to address the question of whether nonhuman animals possess the capacity for episodic memory (for excellent discussions of this issue see Aggleton and Pearce, 2001; Morris, 2001). Tulving (2002) proposed that a key feature of episodic memory is "conscious recollection" that is experienced as a form of "mental time travel." However, it is not clear to us that these mental faculties are, in fact, a necessary prerequisite for a memory system, which supports the ability to recall personally experienced events or how this could be experimentally demonstrated. We leave it to others to debate whether rodents possess the capacity for consciousness and mental time travel. In any case, the history of psychology is replete with examples of "uniquely human" cognitive functions, which were later demonstrated in so-called lower animals. Given the remarkable homology of mammalian nervous systems and the fact that the ability to explicitly recall previous experiences has such obvious adaptive value, we suggest that, in the absence of contradictory evidence, the most conservative position is to assume that rodents possess an episodic memory system that is qualitatively similar to that of humans.

The relationship between hippocampal place fields and the spatial geometry of the environment has been extensively documented (e.g., Kubie and Ranck, 1983; Muller and Kubie, 1987b; O'Keefe and Burgess, 1996a; Gothard et al., 1996). The spatial layout of an environment is a critical feature of any context and several authors have used the term "spatial context" to denote this relationship (Nadel et al., 1985; Mizumori et al., 1999; Jeffery et al., 2004). It has been suggested that spatial context coding is one example of the general context processing function of the hippocampus (Mizumori et al., in press; Smith et al., 2004; Smith and Mizumori, 2006). Indeed, the spatial representations seen in our studies were clearly dependent on nonspatial features of the context (Fig. 1). Place fields were observed but their expression was highly dependent on the current context, as defined by the task demands, suggesting that spatial coding is subordinate to context processing. This idea is consistent with other findings of striking changes in place fields in response to changes in nongeometric features of the task, such as colors and odors (Anderson and Jeffery, 2003; Hayman et al., 2003), task demands and reward conditions (Fig. 1, Markus et al., 1995; Smith and Mizumori, 2006), and problem solving strategy (Yeshenko et al., 2001). 
Previous authors have debated the issue of whether spatial information has a special status as far as hippocampal processing is concerned (Nadel and Eichenbaum, 1999; O'Keefe, 1999; Shapiro and Eichenbaum, 1999). The spatial firing properties of hippocampal neurons are so striking that even a casual observer of a recording session can easily identify the place field of a well isolated neuron and place fields are reliably observed whenever subjects are allowed to explore an environment, suggesting a special status of spatial information. However, the fact that place fields are a predominant correlate of hippocampal neurons may simply be due to the fact that spatial geometry and context are highly correlated. New places are very likely to be new contexts, with new behavioral and cognitive demands. It is suggested here that a primary function of the hippocampus is to differentiate contexts. Therefore, hippocampal neurons should be responsive to any information that provides an effective means of identifying the context. Spatial information may serve this purpose so reliably that its inclusion in context representations is largely automatic. However, other kinds of information can also serve this purpose. As an example, for many species, olfactory information is particularly useful in distinguishing contexts. Consistent with this idea, hippocampal neurons are highly responsive to odor cues (Eichenbaum et al., 1987; Eichenbaum, 1998). In fact, experimental context manipulations frequently involve changes in the specific odors present in the learning situation (Hall and Honey, 1989; Penick and Solomon, 1991; Freeman et al., 1997). Thus, although hippocampal neurons may encode olfactory information, like the encoding of spatial information, this may occur in the service of encoding contexts. Consistent with this idea, hippocampal neurons respond to virtually any potentially important stimulus (Solomon et al., 1986; e.g., Eichenbaum et al., 1987; Kang and Gabriel, 1998; Moita et al., 2003).

\section{THE ROLE OF VOLUNTARY MOVEMENT IN} SPATIAL CODING

The fact that spatial representations have been reported as the predominant form of representations in the hippocampus may be due, in part, to the way in which place cell experiments with rodents are structured. These experiments invariably involve exploration of the environment by subjects. Active exploration involving voluntary movement through the environment may bias the nature of organization of information within the hippocampus. Voluntary movement may direct spatial and movement signals through the medial EC such that networks of spatial grid cells become activated (Hafting et al., 2005; Leutgeb et al., 2005; Sargolini et al., 2006). Grid cells selectively discharge when rats traverse positions that coincide with the vertices of a triangular tessellating grid that spans the environment. The grid fields appear to represent conjunctions of location, direction, and movement information within a two-dimensional coordinate representation of the environment. Thus, the EC may pass to the hippocampus a spatial framework within which to organize and process context-specific in- formation. Voluntary movement, then, may predispose a significant number of hippocampal neurons to organize contextdefining information in the form of location-selective firing. This account of the role of voluntary movement in hippocampal neural codes is consistent with the observation that place fields are not observed, or are not as robust, when subjects are passively transported through the environment rather than when they are allowed to actively explore (Foster et al., 1989; Gavrilov et al., 1998). Also consistent is the finding that hippocampal place fields are observed upon first exposure to a new environment (Muller and Kubie, 1987a; Wilson and McNaughton, 1993; Markus et al., 1995; O'Keefe and Burgess, 1996b; Hetherington and Shapiro, 1997; Frank et al., 2004). The role for experience may be to define, and then adjust the specificity and reliability of the place field to more accurately reflect the learned significance of the context. It remains to be determined whether properties of entorhinal cortical grid fields are impacted by learning as well.

The tendency for voluntary navigation to impose a spatial organization of contextual information may also explain why place fields have not been seen as the predominant form of coding in the primate hippocampus. Whereas rodents explore the environment by active locomotion, primates accomplish much of their exploration visually, by directing their gaze about the environment. Consistent with this, the hippocampal neurons of monkeys respond primarily when the subject directs its gaze at a particular part of the environment (Rolls, 1999). Thus, the apparent differences between primates and rodents may not indicate fundamentally different computations by the hippocampus, but rather that they may result from difference in the kinds of information that are input to the hippocampus by the attentional systems of the brain.

Active exploration is known to modulate the firing of hippocampal interneurons (Ranck, 1973). As is the case for place fields, the movement related firing of hippocampal interneurons has also been shown to be context-dependent (Yeshenko et al., 2004). Therefore, the interneuron population may represent context-appropriate response information. Ultimately, then, context discrimination involves network interactions between pyramidal neurons and interneurons.

Place cells exhibit characteristic short-lasting, high frequency bursts of action potentials when rats pass through the neuron's place field (Ranck, 1973). This type of phasic, burst firing pattern may be associated with synaptic plasticity using long-term potentiation-like mechanisms, possibly resulting in the encoding of discrete features of a situation that do not change very rapidly or often (e.g., significant locations, reward expectations, task phase). Interneurons, on the other hand, continuously discharge at high rates, a pattern that is well suited to encoding rapidly and continuously changing features (e.g., changes in movement and orientation during task performance). Thus, the specific contribution of pyramidal neurons and interneurons to context discrimination may differ in large part because of their different intrinsic cellular properties. The complex combination of features encoded by both pyramidal neurons and interneurons, then, provides hippocampal computational networks with 
a rich array of information with which to identify and distinguish unique situations or contexts.

\section{A ROLE FOR PLACE FIELDS IN CONTEXT DISCRIMINATION}

There are many reports of place fields that rapidly reorganize (i.e., change field location and/or firing rate) when the environment changes, while other place fields persist despite changes in contextual features (Mizumori et al., 1999; Lee et al., 2004; Leutgeb et al., 2004). The responsive place fields may reflect the current contextual features while the persistent fields may reflect expected contextual features. If the current context is determined to be different from the expected context, then an appropriately changed message may be sent to update cortical memory circuits, which in turn will ultimately update the most recent hippocampal expectation for a context. The latter process should result in a subsequent reorganization of neuronal activity patterns in hippocampus. If a context is defined by a unique array of inputs, then, in theory, a change in any one or combination of features should produce an "error" signal that reflects a mismatch (Vinogradova, 1995; Mizumori and Leutgeb, 1999; Anderson and Jeffery, 2003; Hasselmo and McGaughy, 2004; Jeffery et al., 2004; Treves, 2004; Hasselmo, 2005a,b; Leutgeb et al., 2005). If the result of match-mismatch operations indicate that a context has not changed (i.e., there is no place field reorganization), a consistent hippocampal output will result in the persistence of currently active neural activity patterns. As a result, the most recently engaged memory system and behavioral expression patterns will be maintained.

\section{HIPPOCAMPAL NEURONAL RESPONSES} DIFFERENTIATE CONTEXTS BUT NOT EPISODIC MEMORIES OF INDIVIDUAL TRIALS

Examination of the spatial and event related neuronal responses recorded during individual training trials also suggested a hippocampal role in differentiating contexts. Figure 2 illustrates examples of neuronal spike trains emitted when the rats entered a place field or experienced task relevant events. The spike trains were remarkably different in the two contexts, but they were quite similar from one trial to the next. For example, the neurons in Figures $2 \mathrm{~A}$ and $\mathrm{B}$ fired similarly upon the rats' entry into the place field in one context, but fired very little during passes through the same region of space in the other context. The responses to the reward and the return of the rat to the ITI platform after training trials were also similar from one trial to the next, but quite different across contexts (Figs. 2C-E).

Since the neuronal response patterns were relatively similar from one trial to the next, they are more closely associated with differentiating contexts than differentiating individual trials from each other. These data provide additional support for the idea that hippocampal neuronal response patterns could serve

\section{Context A Context B}

A.
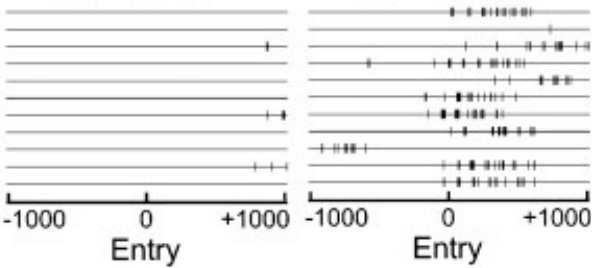

B.
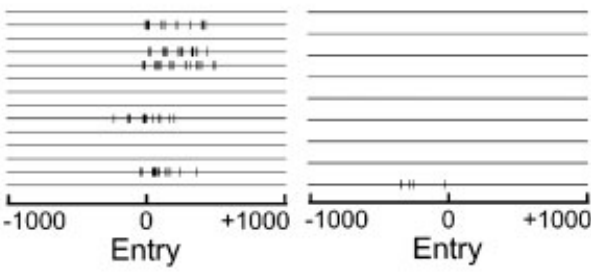

C.
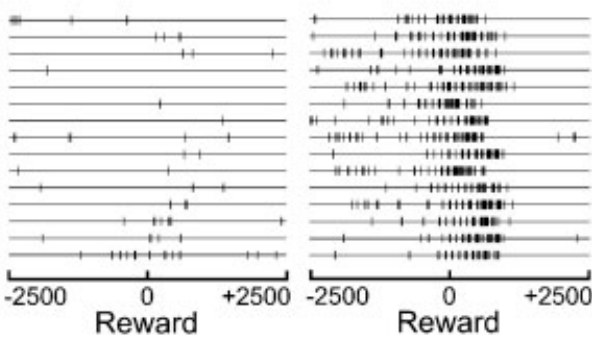

D.
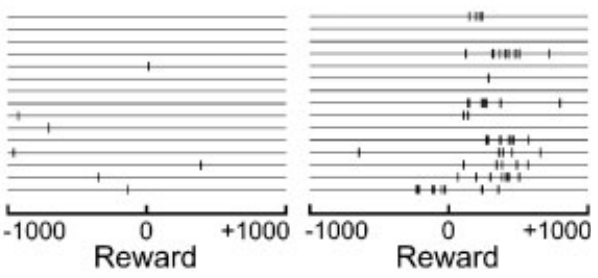

E.
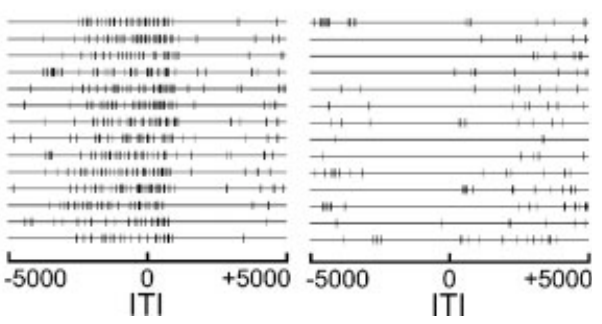

FIGURE 2. Spike trains emitted by five different neurons on individual training trials. Each pair of the plots illustrates the firing of a different neuron during the first half of the session (Context A) when the reward was always placed on the east arm, and during the second half (Context $B$ ) when the reward was always placed on the west arm. The plots illustrate neuronal firing at the time of entry into a place field (plots A and B), the reward (C-D) and arrival at the intertrial interval (ITI) platform after training trials. Each trial is represented by a horizontal line and each tick mark represents a single spike. The time relative to the event is given in milliseconds. The spike trains were relatively similar from one trial to the next within a particular context, but differed markedly between the two contexts.

to differentiate contexts. However, these observations also have important implications for episodic memory accounts of hippocampal function. A key feature of the episodic memory system is that it allows subjects to distinguish one episode from other 

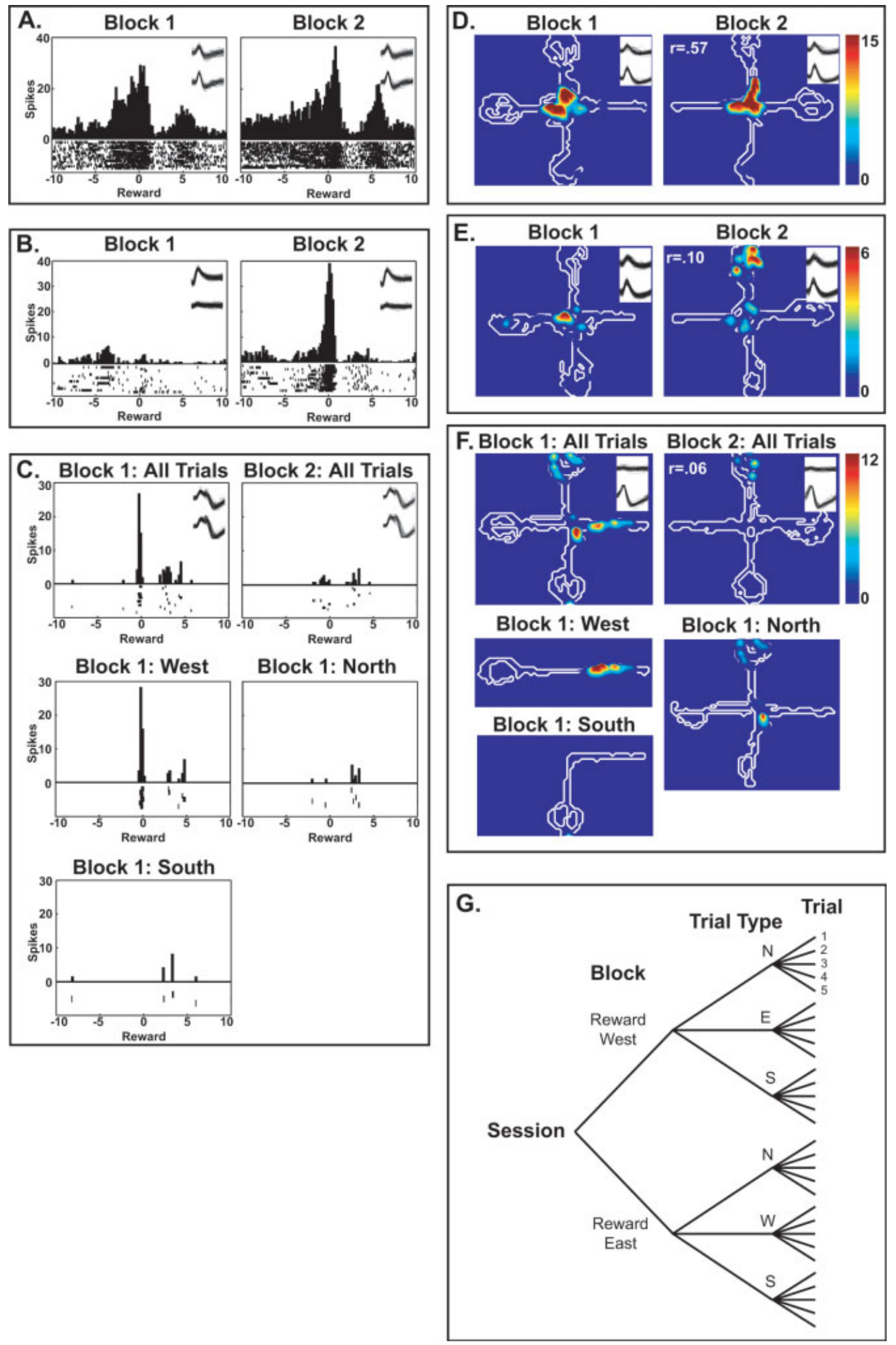

FIGURE 3 (Overleaf) 
similar episodes. Because the neuronal responses were similar from one trial to the next, they are not a good candidate mechanism for differentiating one trial from another. Yet, rats can readily distinguish brief episodes like an individual training trial if it is necessary to do so to perform a task correctly. For example, they can learn spatial alternation tasks, which require subjects to remember the reward location on the previous trial so they can select the opposite location on the following trial (Aggleton et al., 1986; Wood et al., 2000). Presumably then, rats can form episodic memories of individual training trials. However, the spatial and event-related neuronal responses reported here do not account for this ability. To our knowledge, neurophysiological responses that could reliably differentiate one trial from subsequent similar trials have not been reported.

\section{COULD EPISODIC MEMORY IMPAIRMENTS RESULT FROM CONTEXT PROCESSING DEFICITS?}

Although a growing body of data supports the context processing account of hippocampal function, impairment of episodic memory is a well-documented consequence of hippocampal damage in humans (Vargha-Khadem et al., 1997; Tulving and Markowitsch, 1998; Rosenbaum et al., 2005), and the effects of lesions in animals are consistent with this idea (Agster et al., 2002; Ergorul and Eichenbaum, 2004). One possible explanation is that these episodic memory impairments are secondary to context processing deficits. Episodic memory, by definition, involves the encoding of the time and place where the event or episode occurred. Thus, contextual information is a necessary prerequisite for episodic memories. It follows then, that the loss of hippocampal context processing would result in impaired episodic memory functions. Consistent with this idea, several recent reports indicate that human subjects with hippocampal damage are impaired in processing contextual information (Chun and Phelps, 1999; Weis et al., 2004; LaBar and Phelps, 2005; Shanks et al., 2005). The hippocampus may contribute contextual information to an extended circuitry, which includes, but is not limited to, the hippocampus. Several authors have suggested that hippocampal memory functions are mediated by circuitry involving the EC, anterior thalamus, prefrontal cortex, and retrosplenial cortex (Aggleton and Brown, 1999; Eichenbaum, 2000; Suzuki and Eichenbaum, 2000; Smith et al., 2004; Wiltgen et al., 2004; Siapas et al., 2005).

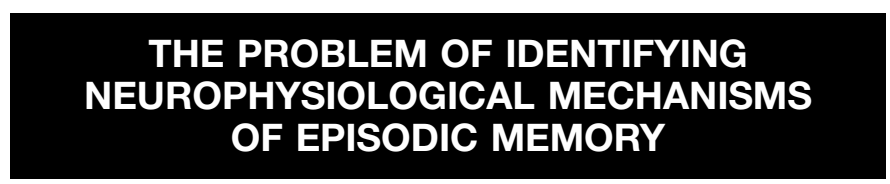

The episodic memory refinement of the declarative memory theory presents new challenges for proponents whose approach involves measuring brain activity, from single unit activity to functional magnetic resonance imaging. Because a key feature of episodic memory is the ability to distinguish one event from another, it is now incumbent on proponents of the episodic memory account of hippocampal function to show that hippocampal neuronal activity can distinguish one event from other similar events.

How might the brain represent distinct episodic memories? We have proposed that contextual information can be represented by neuronal response patterns distributed across a population of neurons. Similarly, individual episodic memories could be represented by neuronal response patterns. However, if an episodic memory must differentiate one experience from all others, then the pattern of neural responses that represents that experience would have to be unique, never to occur again except during the retrieval of that specific memory. If this is the case, the identification of these neuronal response patterns presents some formidable experimental difficulties.

One approach would be to record neuronal responses while subjects are exposed to a unique experience and then to show that the same neuronal response pattern recurs during the subsequent retrieval of that specific memory but not during similar experiences, which should be encoded as new episodic memories. To accomplish this, one would need to induce memory retrieval without requiring the subject to perform another trial,
FIGURE 3. Hippocampal neurons differentiated contexts at various levels of specificity. Some neurons exhibited similar reward responses or place fields throughout the session, regardless of the reward location or the start position (plots $A$ and D). Other neurons responded differentially in the two contexts, as defined by the location of the reward, which was always placed on the east arm during Block 1 and west arm during Block 2 (plots B and E). Still other neurons responded only during specific trial types, defined by the start position. In $\mathrm{C}$, plots that included the data of all of the trials, regardless of the start position, show that the neuron exhibited a reward response during the first block (Block 1: All) but not during the second block (Block 2: All). However, when the data are plotted separately according to the start position, it is clear that the neuron only responded during those trials in Block 1 which had started from the west arm (Block 1: West) and not during trials that started from the north or south start positions. Similarly, the neuron in plot $F$ exhibited a place field in the first block (Block 1: All) but not during the second block (Block 2: All). However, the field was only present during those trials in Block 1, which had started from the west arm (Block 1: West) and not during trials that started from the north or south start positions (see also Fig. 1E). These different kinds of responses form a hierarchical coding scheme, which could differentiate contexts at several levels of specificity. As illustrated in G, some neurons responded at the level of the entire session. Within a given session, however, some neurons responded differentially in the two blocks, which were characterized by different reward locations (i.e., the intended context manipulation). Within a given block, some neurons responded differentially according to the trial type as characterized by the start position. These neurons may treat the different trial types as separate contexts since they require differing behavioral responses. Neuronal responses that clearly differentiated one trial from another (the Trial level) were not found. 
which would be a new episode. Wilson and McNaughton (1994) approximated this procedure by showing that neuronal firing patterns observed during task performance were reexpressed during subsequent sleep periods, when subjects may be consolidating task-relevant memories. However, without a behavioral assay that specifically demonstrates that the subject has retrieved the relevant memory, it is difficult to be certain that the subject is actually engaged in mnemonic information processing.

Hippocampal neurons respond to a wide variety of task relevant stimuli and events (Eichenbaum et al., 1987; Kang and Gabriel, 1998; Smith and Mizumori, 2006). A particularly intriguing finding is that hippocampal neurons frequently respond preferentially to conjunctions of events, or places and events (Eichenbaum et al., 1987; Wood et al., 1999; Ferbinteanu and Shapiro, 2003; Moita et al., 2003). Previously, proponents of the declarative memory hypothesis could logically point to such neuronal responses as evidence of the encoding of stimuli, events and the relations among them. Place fields can also be understood in terms of the encoding of the relations among the stimuli that comprise a given environment (Shapiro et al., 1997). This relational coding scheme is a key feature of the declarative memory hypothesis and provides a comprehensive account of a wide range of the observed spatial and nonspatial neuronal responses (Cohen and Eichenbaum, 1994; Eichenbaum and Cohen, 2001).

The context processing account of hippocampal function presented here is essentially in accordance with this idea. However, our recent findings suggest that these conjunctive or relational neuronal responses are not consistent representations of such relations, but rather they depend on the context. For example, neurons that exhibited place fields or reward responses, which were contingent on a particular start location, typically exhibited this conjunctive firing in only one context or the other (Fig. 1E, see also Figs. 3C,F), suggesting that relational coding is subordinate to, or a component of, context coding. Also, the encoding of some kinds of stimulus relations (e.g., that certain events tend to co-occur or that certain events or stimuli are associated with a particular place) is more closely related to semantic memory for facts and general knowledge than episodic memory. Like other hippocampal neuronal responses, these relational responses are similar from one trial to the next, and therefore do not provide a mechanism for distinguishing one experience from another, as required for episodic memory. Thus, the neurophysiological responses that provided convincing support for a hippocampal role in declarative memory, which encompasses both semantic and episodic memory, do not seem adequate to fully account for the more restrictive episodic memory requirements.

\section{THE PROBLEM OF TEMPORAL SEQUENCING} IN EPISODIC MEMORY

Although the above described neuronal responses to events, or conjunctions of events, are not sufficient to support episodic memory by themselves, they may make a critical contribution to episodic memory. A given episode could be represented by a generic representation of an event, such as a reward, along with a temporal metric that would provide chronological information so that any given reward can be distinguished from previous and subsequent rewards. It has been proposed that rhythmic oscillations in the EEG could provide such a temporal metric (for reviews of this issue, see Hippocampus, 15(7), 2005). A prominent feature of the hippocampus and related structures is rhythmic EEG oscillations in the $7-12 \mathrm{~Hz}$ range, referred to as the theta rhythm. During a pass through a place field, pyramidal neuron spike bursts occur at progressively earlier phases of the theta cycle (O'Keefe and Recce, 1993). Thus, the relationship between the pyramidal neuron firing and the theta phase changes systematically as the rat progresses through the field. This phenomenon, known as phase precession, has been proposed as a potential timing mechanism.

Episodic memory does not necessarily include an absolute temporal metric. It may be sufficient that sequential order is preserved such that subjects can remember that certain episodes preceded or followed others. Neuronal activity synchronized to the theta rhythm may provide a mechanism by which different neurons that fire in a particular sequence become associated (Buzsaki, 2005). When a rat travels through a series of place fields along a given path through the environment, spike timing dependent plasticity would associate each location with previous and upcoming locations, thereby preserving sequential information. As mentioned, the firing patterns observed while rats run on a maze are 'replayed' during subsequent sleep periods (Wilson and McNaughton, 1994). Consistent with the encoding of sequences, the firing order of the neurons observed during task performance was preserved during sleep.

The findings discussed above may represent important initial steps of understanding the neurophysiological mechanisms of temporal processing in the hippocampus. However, it is difficult to reconcile the very brief time scales associated with the theta rhythm with the time scales relevant to episodic memory. The events that comprise a given episodic memory can transpire over the course of minutes or hours and humans routinely remember the order of occurrence of various memory episodes that span decades. Also, the encoding of a sequence of locations along a path traversed by a rat may be superficially similar to remembering a particular sequence of events that comprise an episodic memory. However, it is not clear that when a rat remembers a specific trial, the memory is in the form of a highly structured list of sequentially occupied spatial positions. Human episodic memory seems qualitatively different from the kind of moment by moment structure represented by EEG sequencing mechanisms. Finally, although these mechanisms may explain the encoding of the sequence of events (or positions) that comprise a given pass or trial, they do not by themselves provide a means of distinguishing one trial from another. These issues will need to be resolved before the temporal properties of neuronal activity can account for the chronology of episodic memory. 


\section{A HIERARCHICAL CODING SCHEME FOR CONTEXT DIFFERENTIATION}

The problem of differentiating individual memory episodes from each other is similar to the problem of differentiating individual contexts. Findings of highly differentiated neuronal firing patterns in various situations have led us to think of hippocampal neuronal responses in terms of their ability to differentiate among these situations. Intriguingly, hippocampal neurons seem to differentiate contexts at various levels of specificity (Fig. 3). Neurons at the most general level responded to the reward throughout the training session, regardless of the reward location. Neurons at the next level of specificity responded differentially, depending on the reward location. These are the responses we have described above as 'context specific' because they were sensitive to our intended context manipulation. However, other neurons responded with an even greater degree of specificity. These neurons responded to the reward only when it was presented at a particular location and when the rat had arrived at that location from a particular start position. For example, the neuron in Figure 3C responded during the first half of the training session, when the reward was located on the east arm, but only when the rat had arrived at that location from the west arm. Neurons with place fields exhibited similar varying degrees of specificity. Some neurons exhibited the same place field regardless of the reward location. Others exhibited a field only when the reward was located on the east or west arm. Still others exhibited place fields only when the rat had arrived at the field from a particular start location. Examples of these responses are shown in Figures 1E and 3F.

These responses form a hierarchical coding scheme in which each kind of response represents a subset of the responses at the next highest level of coding. These different kinds of responses could support progressively finer levels of context differentiation. We intended to manipulate the context by changing the reward location. This manipulation was effective insofar as many neurons responded differentially in the two session halves, which were characterized by different reward locations. However, the rats may have spontaneously differentiated "contexts" at a finer resolution, according to the start position for each trial. Jeffery (2004) observed that different kinds of trials involving various trajectories may constitute different contexts. Consistent with this idea, the neurons that exhibited start position-specific firing patterns in our studies may have treated the set of trials that began from a given start position as a unique context, distinct from those trials that began from other start positions.

Previous studies have shown that place fields can be dependent on the start or goal positions (Frank et al., 2000; Wood et al., 2000; Ferbinteanu and Shapiro, 2003). Our data illustrate that these responses represent one of several possible levels of differentiation since the trajectory-dependent firing was context specific. Moreover, the relative importance of each level of differentiation may be indicated by the percentage of the neu- ronal population dedicated to discriminating at that level. Because our experimental design involved different response requirements in the two blocks of trials that comprised each training session, the ability to differentiate blocks was probably the most important level of context discrimination. Consistent with this idea, most of the neurons responded differentially in the two blocks of each training session $(75.6 \%$ of the neurons with reward responses and $76.3 \%$ of the neurons with place fields).

Neuronal responses that differentiate trial types (on the basis of the start position) could also provide important information that prepares the subject for different behavioral responses. Trials that began from the north start position required one response (e.g., right turn) whereas trials that began from the south position required a different response (e.g., left turn). The need for this level of differentiation is even more apparent in spatial alternation tasks, where the only requirement is that the rat must explicitly distinguish those trials, which require a right turn response from those that require a left turn response. Under these conditions, $67 \%$ of the hippocampal neurons differentiated left turn trials from right turn trials (Wood et al., 2000). In our studies, differentiating trial types may have been relatively less important than differentiating blocks. Consistent with this idea, a smaller percentage of the neurons differentiated trial types in our studies $(12.8 \%$ of the neurons with reward responses and $15.3 \%$ of the neurons with place fields). Only $11.5 \%$ of the neurons with reward responses and $8.4 \%$ of the neurons with place fields responded similarly throughout the session.

Progressively finer levels of differentiation may be critically important for distinguishing different episodes that occur in similar contexts. A hierarchical organization of neural representations may identify a set of rewards, which occurred on certain types of trials, which were a subset of the rewards that occurred during a specific block of trials, and which were a subset of the rewards that occurred during a specific training session. The combination of these different levels of processing approximates the ability to remember a specific reward. Clear neuronal responses at the finest level of detail (i.e., the individual trial level) were not observed in our data. However, the addition of temporal information to the above described hierarchy could contribute to an animal's ability to distinguish trials, and could therefore support episodic memory for individual training trials.

\section{CONCLUSIONS}

Increasingly, evidence from different laboratories is converging on the view that the hippocampus serves to process contextual information. Our data suggest that place fields are part of a neural representation of the context. The hippocampus encodes context-relevant information at many levels of complexity, perhaps according to a hierarchical organization. While place fields are exquisitely responsive to changes in the context, we 
also show that nonspatial representations are sensitive to changes in the context. Therefore, it is suggested that the spatial information is but one of the many kinds of information that serves the general context processing function of the hippocampus. An important implication of the proposed context discrimination hypothesis is that the formation and retention of episodic memories involves a neural system that extends beyond hippocampus.

\section{REFERENCES}

Aggleton JP, Brown MW. 1999. Episodic memory, amnesia, and the hippocampal-thalamic axis. Behav Brain Sci 22:425-444, discussion 444-489.

Aggleton JP, Pearce JM. 2001. Neural systems underlying episodic memory: Insights from animal research. Philos Trans R Soc Lond B Biol Sci 356:1467-1482.

Aggleton JP, Hunt PR, Rawlins JN. 1986. The effects of hippocampal lesions upon spatial and non-spatial tests of working memory. Behav Brain Res 19:133-146.

Agster KL, Fortin NJ, Eichenbaum H. 2002. The hippocampus and disambiguation of overlapping sequences. J Neurosci 22:57605768.

Anagnostaras SG, Gale GD, Fanselow MS. 2001. Hippocampus and contextual fear conditioning: Recent controversies and advances. Hippocampus 11:8-17.

Anderson MI, Jeffery KJ. 2003. Heterogeneous modulation of place cell firing by changes in context. J Neurosci 23:8827-8835.

Balaz MA, Capra S, Hartl P, Miller RR. 1980. Contextual potentiation of acquired behavior after developing direct context-US associations. Learn Mem 12:383-397.

Blanchard DC, Blanchard RJ. 1972. Innate and conditioned reactions to threat in rats with amygdaloid lesions. J Comp Physiol Psychol 81:281-290.

Buckmaster CA, Eichenbaum H, Amaral DG, Suzuki WA, Rapp PR. 2004. Entorhinal cortex lesions disrupt the relational organization of memory in monkeys. J Neurosci 24:9811-9825.

Bunsey M, Eichenbaum H. 1996. Conservation of hippocampal memory function in rats and humans. Nature 379:255-257.

Buzsaki G. 2005. $\theta$ Rhythm of navigation: Link between path integration and landmark navigation, episodic and semantic memory. Hippocampus 15:827-840.

Chun MM, Phelps EA. 1999. Memory deficits for implicit contextual information in amnesic subjects with hippocampal damage. Nat Neurosci 2:844-847.

Cohen NJ, Eichenbaum H. 1994. Memory, Amnesia, and the Hippocampal System. Cambridge: MIT Press.

Eichenbaum H. 1998. Using olfaction to study memory. Ann NY Acad Sci 855:657-669.

Eichenbaum H. 2000. A cortical-hippocampal system for declarative memory. Nat Rev Neurosci 1:41-50.

Eichenbaum H, Cohen NJ. 2001. From Conditioning to Conscious Recollection: Memory Systems of the Brain. New York: Oxford University Press.

Eichenbaum H, Kuperstein M, Fagan A, Nagode J. 1987. Cue-sampling and goal-approach correlates of hippocampal unit activity in rats performing an odor-discrimination task. J Neurosci 7:716732.

Eichenbaum H, Stewart C, Morris RG. 1990. Hippocampal representation in spatial learning. J Neurosci 10:331-339.

Ergorul C, Eichenbaum H. 2004. The hippocampus and memory for "what," "where," and "when." Learn Mem 11:397-405.
Fanselow MS. 1986. Associative vs topographical accounts of the immediate shock-freezing deficit in rats: Implications for the response selection rules governing species-specific defensive reactions. Learn Motiv 17:16-39.

Ferbinteanu J, Shapiro ML. 2003. Prospective and retrospective memory coding in the hippocampus. Neuron 40:1227-1239.

Foster TC, Castro CA, McNaughton BL. 1989. Spatial selectivity of rat hippocampal neurons: Dependence on preparedness for movement. Science 244:1580-1582.

Frank LM, Brown EN, Wilson M. 2000. Trajectory encoding in the hippocampus and entorhinal cortex. Neuron 27:169-178.

Frank LM, Stanley GB, Brown EN. 2004. Hippocampal plasticity across multiple days of exposure to novel environments. J Neurosci 24:7681-7689.

Freeman JH Jr, Weible A, Rossi J, Gabriel M. 1997. Lesions of the entorhinal cortex disrupt behavioral and neuronal responses to context change during extinction of discriminative avoidance behavior. Exp Brain Res 115:445-457.

Gabriel M. 1993. Discriminative avoidance learning: A model system. In: Vogt BA, Gabriel M, editors. Neurobiology of Cingulate Cortex and Limbic Thalamus. Boston: Birkhauser. pp 478-523.

Gavrilov VV, Wiener SI, Berthoz A. 1998. Discharge correlates of hippocampal complex spike neurons in behaving rats passively displaced on a mobile robot. Hippocampus 8:475-490.

Godden D, Baddely A. 1975. Context-dependent memory in two natural environments: On land and underwater. Br J Psychol 66:325331.

Gothard KM, Skaggs WE, Moore KM, McNaughton BL. 1996. Binding of hippocampal CA1 neural activity to multiple reference frames in a landmark-based navigation task. J Neurosci 16:823835.

Hafting T, Fyhn M, Molden S, Moser MB, Moser EI. 2005. Microstructure of a spatial map in the entorhinal cortex. Nature 436: 801-806.

Hall G, Honey RC. 1989. Contextual effects in conditioning, latent inhibition, and habituation: Associative and retrieval functions of contextual cues. J Exp Psychol 15:232-241.

Hasselmo ME. 2005a. The role of hippocampal regions CA3 and CA1 in matching entorhinal input with retrieval of associations between objects and context: Theoretical comment on Lee et al. (2005). Behav Neurosci 119:342-345.

Hasselmo ME. 2005b. What is the function of hippocampal $\theta$ rhythm?-Linking behavioral data to phasic properties of field potential and unit recording data. Hippocampus 15:936-949.

Hasselmo ME, McGaughy J. 2004. High acetylcholine levels set circuit dynamics for attention and encoding and low acetylcholine levels set dynamics for consolidation. Prog Brain Res 145:207-231.

Hayman RM, Chakraborty S, Anderson MI, Jeffery KJ. 2003. Context-specific acquisition of location discrimination by hippocampal place cells. Eur J Neurosci 18:2825-2834.

Hetherington PA, Shapiro ML. 1997. Hippocampal place fields are altered by the removal of single visual cues in a distance-dependent manner. Behav Neurosci 111:20-34.

Hirsh R. 1974. The hippocampus and contextual retrieval of information from memory: A theory. Behav Biol 12:421-444.

Jeffery KJ. 2004. Remembrance of futures past. Trends Cogn Sci 8: 197-199.

Jeffery KJ, Anderson MI, Hayman R, Chakraborty S. 2004. A proposed architecture for the neural representation of spatial context. Neurosci Biobehav Rev 28:201-218.

Johnson JD. 2004. Episodic memory and the hippocampus: Another view. Med Hypotheses 63:963-967.

Kang E, Gabriel M. 1998. Hippocampal modulation of cingulo-thalamic neuronal activity and discriminative avoidance learning in rabbits. Hippocampus 8:491-510.

Kennedy PJ, Shapiro ML. 2004. Retrieving memories via internal context requires the hippocampus. J Neurosci 24:6979-6785. 
Kim JJ, Fanselow MS. 1992. Modality-specific retrograde amnesia of fear. Science 256:675-677.

Kubie JL, Ranck JB. 1983. Sensory-behavioral correlates in individual hippocampus neurons in three situations: Space and context. In: Seifert W, editor. The Hippocampus. New York: Academic Press. pp 433-447.

LaBar KS, Phelps EA. 2005. Reinstatement of conditioned fear in humans is context dependent and impaired in amnesia. Behav Neurosci 119:677-686.

Lee I, Yoganarasimha D, Rao G, Knierim JJ. 2004. Comparison of population coherence of place cells in hippocampal subfields CA1 and CA3. Nature 430:456-459.

Leutgeb S, Leutgeb JK, Treves A, Moser MB, Moser EI. 2004. Distinct ensemble codes in hippocampal areas CA3 and CA1. Science 305:1295-1298.

Leutgeb S, Leutgeb JK, Moser MB, Moser EI. 2005. Place cells, spatial maps and the population code for memory. Curr Opin Neurobiol 15:738-746.

Maren S. 2001. Neurobiology of Pavlovian fear conditioning. Annu Rev Neurosci 24:897-931.

Markus EJ, Qin YL, Leonard B, Skaggs WE, McNaughton BL, Barnes CA. 1995. Interactions between location and task affect the spatial and directional firing of hippocampal neurons. J Neurosci 15: 7079-7094.

McNaughton BL, Barnes CA, Gerrard JL, Gothard K, Jung W, Knierim JJ, Kudrimoti H, Skaggs WE, Suster M, Weaver KL. 1996. Deciphering the hippocampal polyglot: The hippocampus as a path integration system. J Exp Biol 199:173-185.

Mishkin M. 1978. Memory in monkeys severely impaired by combined but not by separate removal of amygdala and hippocampus. Nature 273:297-298.

Mizumori SJ, Leutgeb S. 1999. Interpreting neural representations of aged animals. Hippocampus 9:607-608.

Mizumori SJ, Ragozzino KE, Cooper BG, Leutgeb S. 1999. Hippocampal representational organization and spatial context. Hippocampus 9:444-451.

Mizumori SJ, Yeshenko O, Gill KM, Davis DM. 2004. Parallel processing across neural systems: Implications for a multiple memory system hypothesis. Neurobiol Learn Mem 82:278-298.

Mizumori SJY, Smith DM, Puryear CB. Mnemonic contributions of hippocampal place cells. In: Martinez JLJ, Kesner RP, editors. Neurobiology of Learning and Memory, 4th ed. San Diego, CA: Academic Press (in press).

Moita AP, Svetlana R, Zhou Y, LeDoux JE, Blair HT. 2003. Hippocampal place cells acquire location-specific response to the conditioned stimulus during auditory fear conditioning. Neuron 37:485-497.

Morris RG. 2001. Episodic-like memory in animals: Psychological criteria, neural mechanisms and the value of episodic-like tasks to investigate animal models of neurodegenerative disease. Philos Trans R Soc Lond B Biol Sci 356:1453-1465.

Morris RG, Garrud P, Rawlins JM, O’Keefe J. 1982. Place navigation is impaired in rats with hippocampal lesions. Nature 297:681-683.

Muller RU, Kubie JL. 1987a. The effects of changes in the environment on the spatial firing of hippocampal complex-spike cells. J Neurosci 7:1951-1968.

Muller RU, Kubie JL. 1987b. The effects of changes in the environment on the spatial firing of hippocampal complex-spike cells. J Neurosci 7:1951-1968.

Muller RU, Stead M, Pach J. 1996. The hippocampus as a cognitive graph. J Gen Physiol 107:663-694.

Myers CE, Gluck M. 1994. Context, conditioning, and hippocampal rerepresentation in animal learning. Behav Neurosci 108:835-847.

Nadel L, Eichenbaum H. 1999. Introduction to the special issue on place cells. Hippocampus 9:341-345.

Nadel L, Willner J, Kurz EM. 1985. Cognitive maps and environmental context. In: Balsam P, Tomie A, editors. Context and Learning. Hillsdale, NJ: Erlbaum. pp 385-406.
O'Keefe J. 1976. Place units in the hippocampus of the freely moving rat. Exp Neurol 51:78-109.

O'Keefe J. 1999. Do hippocampal pyramidal cells signal non-spatial as well as spatial information? Hippocampus 9:352-364.

O'Keefe J, Burgess N. 1996a. Geometric determinants of the place fields of hippocampal neurons. Nature 381:425-428.

O'Keefe J, Burgess N. 1996b. Spatial and temporal determinants of the hippocampal place cell activity. In: Ono T, McNaughton B, Molotchnikoff S, Rolls E, Nishijo H, editors. Perception, Memory and Emotion: Frontiers in Neuroscience. New York: Pergamon. pp 359-373.

O'Keefe J, Dostrovsky J. 1971. The hippocampus as a spatial map. Preliminary evidence from unit activity in the freely-moving rat. Brain Res 34:171-175.

O'Keefe J, Recce ML. 1993. Phase relationship between hippocampal place units and the EEG $\theta$ rhythm. Hippocampus 3:317-330.

Olton DS, Becker JT, Handelman GE. 1979. Hippocampus, space, and memory. Brain Behav Sci 2:313-365.

O'Mara SM. 1995. Spatially selective firing properties of hippocampal formation neurons in rodents and primates. Prog Neurobiol 45: 253-274.

Penick S, Solomon PR. 1991. Hippocampus, context, and conditioning. Behav Neurosci 105:611-617.

Phillips RG, LeDoux JE. 1992. Differential contribution of amygdala and hippocampus to cued and contextual fear conditioning. Behav Neurosci 106:274-285.

Ranck JB. 1973. Studies on single neurons in dorsal hippocampal formation and septum in unrestrained rats. I. Behavioral correlates and firing repertoires. Exp Neurol 41:461-531.

Rolls E. 1999. Spatial view cells and the representation of place in the primate hippocampus. Hippocampus 9:467-480.

Rosenbaum RS, Winocur G, Moscovitch M. 2001. New views on old memories: Re-evaluating the role of the hippocampal complex. Behav Brain Res 127:183-197.

Rosenbaum RS, Kohler S, Schacter DL, Moscovitch M, Westmacott R, Black SE, Gao F, Tulving E. 2005. The case of K.C.: Contributions of a memory-impaired person to memory theory. Neuropsychologia 43:989-1021.

Sargolini F, Fyhn M, Hafting T, McNaughton BL, Witter MP, Moser MB, Moser EI. 2006. Conjunctive representation of position, direction, and velocity in entorhinal cortex. Science 312:758-762.

Scoville WB, Milner B. 1957. Loss of recent memory after bilateral hippocampal lesions. J Neurochem 20:11-21.

Shanks DR, Channon S, Wilkinson L, Curran HV. 2006. Disruption of sequential priming in organic an pharmacological amnesia: A role for the medial temporal lobes in implicit contextual learning. Neuropsychopharmacology 31:1768-1776.

Shapiro ML, Eichenbaum H. 1999. Hippocampus as a memory map: Synaptic plasticity and memory encoding by hippocampal neurons. Hippocampus 9:365-384.

Shapiro M, Tanila H, Eichenbaum H. 1997. Cues that hippocampal place cells encode: Dynamic and hierarchical representation of local and distal stimuli. Hippocampus 7:624-642.

Siapas AG, Lubenov EV, Wilson MA. 2005. Prefrontal phase locking to hippocampal theta oscillations. Neuron 46:141-151.

Skaggs WE, McNaughton BL. 1998. Spatial firing properties of hippocampal CA1 populations in an environment containing two visually identical regions. J Neurosci 18:8455-8466.

Smith DM, Mizumori SJY. 2006. Learning-related development of context-specific neuronal responses to places and events: The hippocampal role in context processing. J Neurosci 26:3154-3163.

Smith DM, Wakeman D, Patel J, Gabriel M. 2004. Fornix lesions impair context-related cingulothalamic neuronal patterns and concurrent discrimination learning. Behav Neurosci 118:1225-1239.

Solomon PR, Vander Schaaf ER, Thompson RF, Weisz DJ. 1986. Hippocampus and trace conditioning of the rabbit's classically conditioned nictitating membrane response. Behav Neurosci 100: 729-744. 
Song EY, Kim YB, Kim YH, Jung MW. 2005. Role of active movement in place-specific firing of hippocampal neurons. Hippocampus 15:8-17.

Squire LR, Zola-Morgan S. 1991. The medial temporal lobe memory system. Science 253:1380-1386.

Squire LR, Stark CE, Clark RE. 2004. The medial temporal lobe. Annu Rev Neurosci 27:279-306.

Sutherland GR, Kolb B, Wishaw IQ. 1983. A behavioral analysis of spatial localization following electrolytic, kainate- or colchicineinduced damage to the hippocampal formation in the rat. Behav Brain Res 7:133-153.

Suzuki WA, Eichenbaum H. 2000. The neurophysiology of memory. Ann NY Acad Sci 911:175-191.

Treves A. 2004. Computational constraints between retrieving the past and predicting the future, and the CA3-CA1 differentiation. Hippocampus 14:539-556.

Tulving E. 1972. Episodic and semantic memory. In: Tulving E, Donaldson W, editors. Organization of Memory. New York: Academic Press. pp 381-403.

Tulving E. 2002. Episodic memory: From mind to brain. Annu Rev Psychol 53:1-25.

Tulving E, Markowitsch HJ. 1998. Episodic and declarative memory: Role of the hippocampus. Hippocampus 8:198-204.

Vargha-Khadem F, Gadian DG, Watkins KE, Connelly A, Van Paesschen W, Mishkin M. 1997. Differential effects of early hippocampal pathology on episodic and semantic memory. Science 277:376-380.

Vinogradova OS. 1995. Expression, control, and probable functional significance of the neuronal theta-rhythm. Prog Neurobiol 45:523583.

Weis S, Specht K, Klaver P, Tendolkar I, Willmes K, Ruhlmann J, Elger CE, Fernandez G. 2004. Process dissociation between contextual retrieval and item recognition. Neuroreport 15:2729-2733.
Whishaw IQ, Tomie JA. 1997. Perseveration on place reversals in spatial swimming pool tasks: Further evidence for place learning in hippocampal rats. Hippocampus 7:361-370.

Wiener SI. 1996. Spatial, behavioral and sensory correlates of hippocampal CA1 complex spike cell activity: Implications for information processing functions. Prog Neurobiol 49:335-361.

Wilson MA, McNaughton BL. 1993. Dynamics of the hippocampal ensemble code for space [see comments]. Science 261:1055-1058. [Published erratum appears in Science 1994, 264:16].

Wilson A, McNaughton BL. 1994. Reactivation of hippocampal memories during sleep. Science 19:4090-4101.

Wiltgen BJ, Brown RA, Talton LE, Silva AJ. 2004. New circuits for old memories: The role of the neocortex in consolidation. Neuron 44:101-108.

Winocur G, Olds J. 1978. Effects of context manipulation on memory and reversal learning in rats with hippocampal lesions. J Comp Physiol Psychol 92:312-321.

Wood ER, Dudchenko PA, Eichenbaum H. 1999. The global record of memory in hippocampal neuronal activity [see comments]. Nature 397:613-616.

Wood ER, Dudchenko PA, Robitsek RJ, Eichenbaum H. 2000. Hippocampal neurons encode information about different types of memory episodes occurring in the same location. Neuron 27:623633.

Yeshenko O, Guazzelli A, Mizumori SJY. 2001. Differential hippocampal neuronal activity during place or response performance on a t-maze. Soc Neurosci 131 Abstract 953.8.

Yeshenko O, Guazzelli A, Mizumori SJ. 2004. Context-dependent reorganization of spatial and movement representations by simultaneously recorded hippocampal and striatal neurons during performance of allocentric and egocentric tasks. Behav Neurosci 118:751769. 